\title{
El baluarte de la Alcazaba de la Alhambra: un comienzo de su restauración
}

The bulwark of the Alcazaba at the Alhambra: a starting point in its restauration

\author{
Francisco Javier López Martínez ${ }^{\text {a }}$, Teresa Koffler Urbano ${ }^{b}$ \\ ${ }^{a}$ Universidad Católica de Murcia, Murcia, Spain, fjlopez@ucam.edu \\ ${ }^{\mathrm{b}}$ Archaeologist, Granada, Spain, nornaurd@gmail.com
}

\begin{abstract}
The bulwark of the Alcazaba is one of the polyorcetic reforms that the Catholic Monarchs introduced on the Alhambra since the conquest of Granada in 1492, in order to adapt the fortress to pyrobalistic artillery, as well as to stablish a new relationship with the city. In essence, it was built in rammed earth, using brick and masonry fabrics in certain points.

An important section of its parapet was in a high grade of deterioration, as result of the humidity, the loss of mass of the walls, and deformations caused by uncontrolled vegetation. This situation has triggered on different processes that have recently ended up with the restoration of a part of the bulwark.

The restoration works, carried out over 20 months, have been strongly conditioned not only by the location and the layout of the building, that is at the western end of the monumental complex, crowning a steep slope, but to many functional aspects that have been necessary to plan in order to not interfere with the public visit to the Alhambra.

Parallel to the restauration, archaeological works have been developed both at the outer base of the curtain, flank and face of the bulwark and at the inside, with interesting results.

Due to these archaeological and restoration works, it has been possible to stabilize and improve the state of preservation of the northwest section of this significant part of the Alhambra, improving its stratigraphic reading and increasing in a substantial way the knowledge of this edge of the hill.
\end{abstract}

Keywords: Alhambra, bulwark, restauration, rammed earth.

\section{Introducción}

La Alhambra inspiraba seguridad y poder rodeada de una población leal, pero su eficacia defensiva pesaba menos que su ostentación, sobre todo cuando las armas habían evolucionado al mismo tiempo que los problemas y las intrigas.

En 1492, con la toma de la ciudad por los Reyes Católicos, se produjo una inversión de relaciones y significados. Entonces la Alhambra debía convertirse en una fortaleza persuasiva, en un reducto del poder a salvo y vigilante de una población hostil y de un territorio inseguro.
Poco después de entrar en la ciudadela, los reyes cristianos comienzan su esfuerzo por conservar los palacios y mejorar sus defensas.

Los baluartes, las barreras, y las cavas que hundían la fortaleza en el terreno fuera del alcance enemigo, iban imponiéndose frente a la altura como sueño de dominio y deseo de inexpugnabilidad medievales. Todo respondía al auge y paulatina eficacia de un arma que había cambiado, drásticamente, las formas de ataque y defensa, que hacía caer ciudades e imperios otrora 
fuertes. La artillería pirobalística obligaba a poner distancia, reducir altura, aumentar grosores e impedir, en su caso, el asalto; así como a dar una nueva importancia a la geometría.

\section{El baluarte de la Alcazaba y su construc- ción}

El baluarte de la Alcazaba, o "baluarte de la Mezquita de sobre Darro", constituye el más grande de los encargados por los Reyes Católicos a su "maestro mayor de la artillería real", el aragonés Ramiro López. Las obras comenzaron en 1492 y finalizaron en 1497 (Vilar, 2007). La construcción no resulta tan sofisticada como fue Medina del Campo o será Salsas, pero se trata de una obra rotunda y adaptada a las características de su emplazamiento y a las circunstancias de su momento.

Además de ser uno de los complementos defensivos necesarios, en el juego de significados que siempre desempeñó la ciudad áulica, al baluarte de la Alcazaba correspondía uno nuevo: advertir, amenazar a la población sobre las consecuencias de una rebelión.

Aunque el baluarte queda hoy ocultado por el bosque, en su origen debía de aparecer imponente al proyectarse sobre la ciudad encima de un monte desnudo con el río a sus pies y sus armas de fuego dispuestas o insinuadas, esas armas terribles eran patrimonio exclusivo de los conquistadores tal como se estipulaba en la "capitulacion de la toma é entrega de Granada (en el real de su vega, á 25 dias del mes de noviembre de 1491 años" (Fernández, 1886, 2005, p. 423).

En varias obras del siglo XVI, pinturas, dibujos o relieves (Petrus Christus II, Vico, Felipe Bigarny...), el baluarte aparece representado en perspectiva con merlones y formas difíciles de reconocer salvo por la curva; sí resulta reconocible, sin embargo, en la planta de Machuca.

La obra se define, sobre todo, por un muro perimetral construido en tapiería de hormigón. Su altura es de 5 o 7 tapias más un pretil de mam- postería entre machones y verdugadas de ladrillo que se remata con sillares de travertino de Alfacar. La inestabilidad de dicho pretil ha sido el motivo concreto que ha provocado la redacción de un proyecto y la ejecución de unas obras que acaban de finalizar.

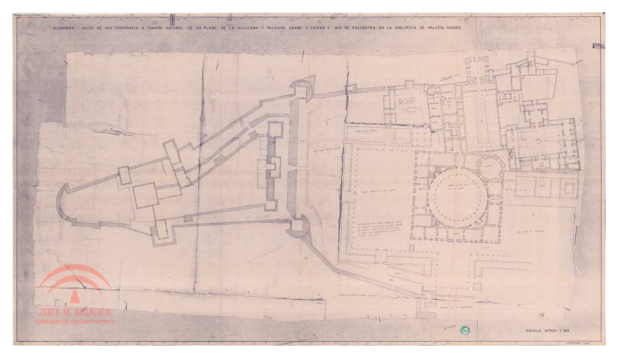

Fig. 1. Planta grande de Machuca: Calco de fotografía de un plano de la Biblioteca del Palacio Real (Madrid), que abarca los Palacios Árabes y de Carlos V y la Alcazaba. APAG, P-008182. http://hdl.handle.net/ $10514 / 4229$.

El espesor de los muros es de, aproximadamente, $2,40 \mathrm{~m}$, de los que unos $0,50 \mathrm{~m}$ corresponden al pretil. En escasos puntos se puede apreciar las huellas de los tapiales, si comparamos las medidas que se evidencian de las fábricas y las huellas en sus paramentos podemos ver que la altura y espesor se relacionan con las dispuestas en el "memorial é condiciones de la obra que se ha de facer en la fortaleza de Hüejar" (AGS, Mar y tierra, leg. 1315; Arántegui, 1891) mientras que la longitud es un tanto desmesurada, en algunos puntos existe la evidencia de un tapial completo incluido el barzón, arrojando una longitud superior a $3 \mathrm{~m}$.

El muro parte, desde el este, de la torre de los Hidalgos y se prolonga casi $38 \mathrm{~m}$ hasta llegar al flanco del baluarte en el extremo oeste de este lienzo o cortina. Este muro arranca directamente del suelo de formación Alhambra hasta discurrir unos $26,5 \mathrm{~m}$, a partir de ese punto el muro baja 2 tapias que se construyeron con ayuda de una zanja practicada en el terreno. De manera que los primeros $26,5 \mathrm{~m}$ presentan 5 tapias de altura más 1 tapia del pretil, y los 11,5 restantes tienen 7 tapias más el pretil; aunque las 2 tapias inferiores están bajo la rasante y son 
más gruesas dando lugar a una zarpa; este arranque más bajo es el que parece continuar por el flanco y la cara del baluarte hacia el oeste, mientras que la coronación del pretil es continua, sin altibajos.

La existencia de estas dos hiladas más bajas responde a la pendiente del terreno, pendiente que se manifiesta en el interior del baluarte.

A lo largo de la cortina (muro norte del conjunto), existen 5 cámaras de tiro con sus correspondientes troneras. Si llamamos $1^{\mathrm{a}}$ a la hilada más baja y las seguimos numerando hacia arriba, resulta que 3 primeras cámaras de tiro con sus troneras se corresponden con la hilada $5^{\mathrm{a}}$, la tronera 4 se encuentra más abajo entre las hiladas $4^{\mathrm{a}}$ y $5^{\mathrm{a}}$, la tronera 5 entre las hiladas $3^{\mathrm{a}} \mathrm{y}$ $4^{\mathrm{a}}$.

A la cortina le sucede el flanco con un ángulo fijante de 100 grados, ángulo característico para el buen funcionamiento de los defensores; pegada al ángulo se halla la tronera 6 encajada en la $4^{\text {a }}$ hilada y dispuesta para el tiro de enfilada. Al dar la vuelta y continuar por la cara se encuentra la tronera 7 construida en la $3^{\mathrm{a}}$ hilada. Después, recorriendo todo el frente curvo del baluarte, se suceden otras 5 troneras hasta llegar a un muro perpendicular que constituye un nuevo flanco.

En su origen, el baluarte y la cortina norte se reducían a un adarve continuo y una línea baja de tiro formada por 12 troneras que recuerdan la barrera de Niebla, pero en algún momento, todavía no precisado, se debió rellenar en su totalidad, o en gran parte, para disponer de una extensa plataforma artillera concebida para grandes piezas (una circunstancia que pudo justificar esta potente reforma pudo ser la ocupación francesa de la Alhambra de 1810 a 1812, pero algunos detalles permiten dudarlo). El gran relleno de tierra se construyó con estribos interiores unidos por un muro perpendicular a todos ellos, formando un peine. Las tierras llegaban hasta la coronación de los estribos, que ahora se hallan a la vista, sobre esa gran masa estribada se construyó una losa de hormigón con un espe- sor superior a los $70 \mathrm{~cm}$ que enrasa con la superficie del adarve. Actualmente el borde de esa losa dibuja una línea sensiblemente paralela a la cara curva del baluarte aumentando unos $5 \mathrm{~m}$ la anchura de lo que fue el adarve original, pero no sabemos si llegó a ocupar un área mayor. Hoy, debido al vaciado de las tierras bajo la losa, ésta ha quedado apoyada sólo en los estribos, aunque cuando se construyó estaba apoyada en la totalidad de su superficie, lo cual representa una situación estructural anómala.

$\mathrm{El}$ pretil tiene un espesor próximo a los 0,50 m y una altura de una tapia, es continuo y está coronado por sillares de caras verticales por el interior y curvas en cuarto de círculo hacia el exterior. Con la forma actual no puede desempeñar con eficacia la protección de un posible tirador, puesto que siempre estaría al descubierto.

Las cámaras de tiro se construyen, con la altura interior de una tapia, como prismas de fábrica de ladrillo cubiertos por una bóveda de cañón muy rebajada y con la directriz inclinada (más alta hacia el interior y más baja hacia la abertura de disparo), se debieron levantar al mismo tiempo que las correspondientes hiladas de tapia pero aquélla fábrica de ladrillo no llegaba hasta la cara exterior, sino que se paraba al faltar la anchura de un tizón para, una vez asentada la pieza de piedra que constituye la tronera (de palo y orbe en nuestro caso), terminarla con el medio pie de ladrillo que aún faltaba. Ese medio pie de ladrillo está casi sin trabar con el resto de la cámara, esta manera de proceder puede tener varias razones: por una parte, dar mayor independencia a los oficios $\mathrm{y}$, por otra, hacer más fácil cualquier reparación en caso de rotura.

\section{Estado de conservación antes de las obras}

La vegetación proliferaba, de una forma relativamente descontrolada, tanto en el entorno inmediato, como en el interior del baluarte o sobre sus estructuras. El agua atravesaba casi constantemente el recinto y salía a través de la tronera 5 , esta salida de agua mantenía húmedo el muro y socavaba su base y paramento. El agua parecía llegar a un depósito o aljibe situado bajo la esquina del baluarte. 


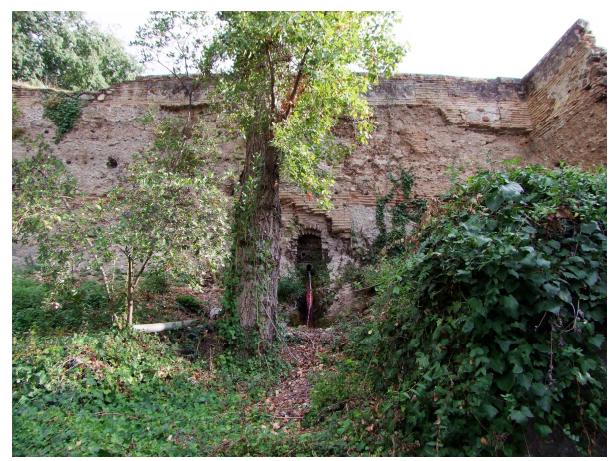

Fig. 2. Aspecto de la zona más deteriorada. Tronera 5 y salida del agua. Encuentro de la cortina con el flanco (Francisco Javier López Martínez, 2013).

Los muros se encontraban con graves pérdidas de material que, de forma gradual, aumentaban hacia el oeste, habiéndose producido las mayores pérdidas en la zona de la tronera 5 y el flanco contiguo.

Tanto el adarve del muro norte, como la plataforma artillera se hallaban cubiertos por vegetación, incluso árboles. El pretil se hallaba deformado, empujado hacia fuera por una raíz situada, aproximadamente en la vertical de la tronera 5, que actuaba como cuña entre el adarve y el pretil. La parte superior del pretil se había perdido en un tramo de 4,70 m. Un gran tramo de pretil se hallaba volado por el desplazamiento experimentado y por la falta de muro que lo sustentara. En el flanco había un tramo de pretil, de unos 2,50 m, desplomado y con deformaciones, a continuación se habían perdido sendos tramos a cada lado de la esquina.

No se sabía cómo era la base de la cortina ni cuál era la relación del baluarte con el aljibe situado a sus pies.

\section{Planteamiento del proyecto}

El proyecto debía de intervenir en esa zona crítica del baluarte. Se trataba de corregir los problemas planteados, muy especialmente el peligro de desplome del pretil, como ya había ocurrido en época indeterminada.

-Dar una salida encauzada al agua para que no siguiera deteriorando las estructuras.
-Recobrar el espesor de los muros para que el pretil tuviera apoyo.

-Limpiar, consolidar y mejorar la lectura de los muros. Limpiar y proteger el adarve.

-Mejorar el conocimiento sobre el baluarte para acercarse a su restauración.

-Posibilitar un acceso al baluarte sin interferir las visitas al conjunto monumental.

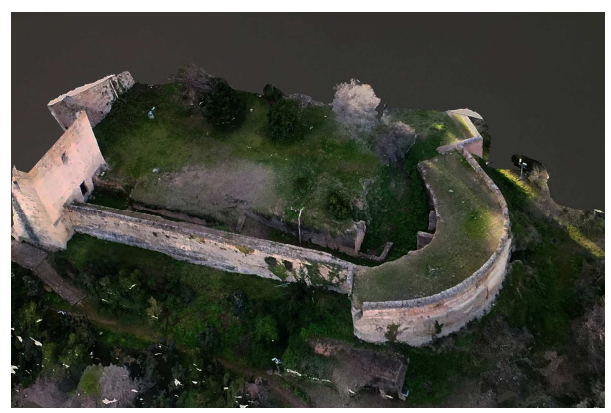

Fig. 3. Modelo del baluarte y la zona de actuación (Antonio Puertas Contreras, 2018).

\section{Las obras}

Las primeras operaciones consistieron en la gestión y disposición de medidas para poder acceder directamente desde la Cuesta de Gomérez, para lo cual, además de conseguir entrar por un callejón cerrado junto al palacio de Los Marqueses de Cartagena fue necesario montar una rampa que salvara un desnivel de 4,5 $\mathrm{m}$ para llegar a un camino en el bosque de la Alhambra de gran pendiente.

Una vez llegados a la zona a intervenir, la instalación de una escalera permitía asaltar el baluarte.

Antes de proceder a los trabajos por el exterior fue necesario apuntalar las zonas más deformadas del pretil, tratando de que el apuntalamiento no impidiera la continuación de los trabajos. Así como la reconducción provisional de las aguas hasta el aljibe evitando pérdidas y humedades.

Se recuperó el suelo del adarve hasta llegar a un gran roto en el extremo oeste, que se decidió respetar como vacío para no cerrar posibilidades de interpretación. 


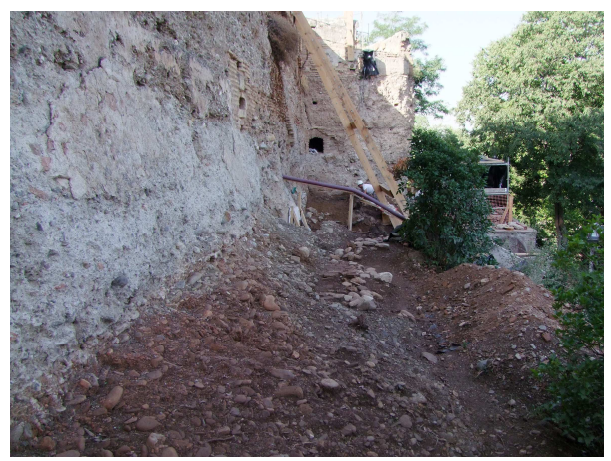

Fig. 4. Aspecto de la zona exterior con los grandes puntales y la conducción provisional del agua a través de la tronera 5 (Francisco Javier López Martínez, 2018).

Se repusieron las piezas de coronación perdidas, con piedra semejante a la original.

Hasta la tronera 4 se hicieron pequeñas intervenciones en el muro para mejorar su conservación y lectura.

Se descubrieron las cámaras de tiro cegadas y se rehicieron las troneras para sujetar zonas de muro con peligro de derrumbes.

En la zona oeste, la más deteriorada, se procedió a rehacer los muros desde su base. Tratando de respetar las juntas y el sentido original de ejecución. Con cada hilada de tapia se preparaba la base para la tronera correspondiente. Las grandes pérdidas sufridas en el suelo de la cámara 4, por donde salía el agua, nos permitió encauzarla de una forma discreta ya que la nueva tubería pudo quedar embebida en la parte de muro que había que reponer (tanto el tramo sensiblemente horizontal como el codo y el tramo vertical).

Las vicisitudes de la obra original aumentaron la complejidad de las obras de restauración,

por ejemplo, originalmente se debió trabajar simultáneamente en dos sentidos: de este a oeste y viceversa, incluso el material correspondiente a cada tajo parecía variar ligeramente de color. La zona más conflictiva era la confluencia de los dos sentidos.

Una cuestión importante fue resolver la esquina, el ángulo formado por el flanco y la cara, en ese punto había más dudas que certezas: ¿Por qué la plataforma estaba achaflanada? ¿Por qué el aljibe parecía meterse dentro del baluarte? La decisión fue consolidar la zona sin reconstruirla y dejar que las tapias se fueran aproximando. Así la esquina se quedaba como pregunta.

La construcción de la $7^{\text {a }}$ hilada supuso alcanzar una de meta: sujetar el pretil desde su base.

En el flanco, hubo que desmontar un trozo de pretil para reconstruirlo en su posición original, pegado a la losa a la que, al parecer, sirvió de encofrado.

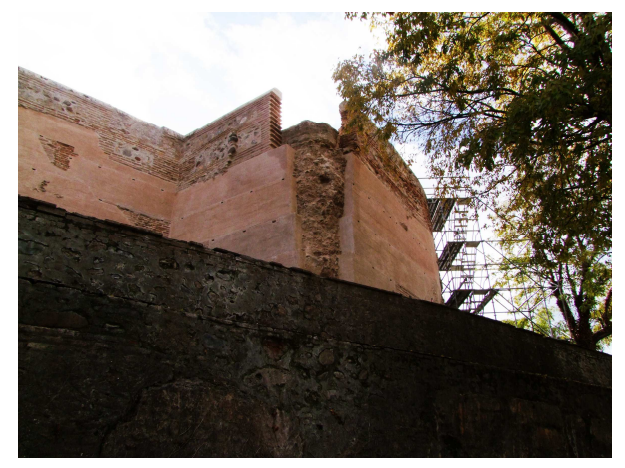

Fig. 5. La esquina del baluarte sobre el aljibe (Francisco Javier López Martínez, 2019).

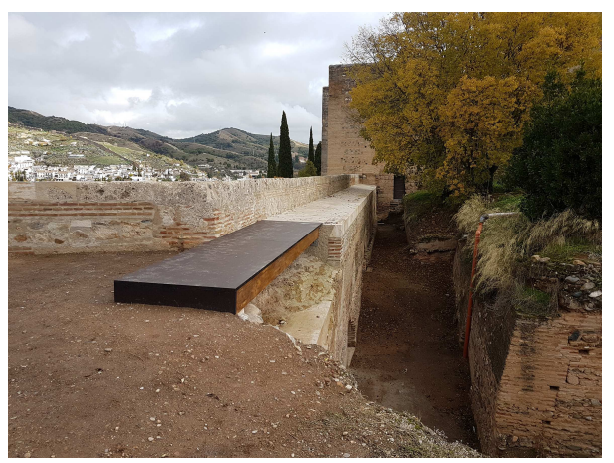

Fig. 6. El adarve y el flanco vistos desde el interior (Francisco Javier López Martínez, 2019).

Consecuentemente con la decisión de no rehacer la esquina, el pretil también quedó interrumpido.

Por último, el tramo de adarve sin rellenar se salvó con un puente que permite el paso al mismo tiempo que observar los restos. 
6. La intervención arqueológica en el Baluarte de la Alcazaba. Resultados preliminares

La intervención arqueológica en el Baluarte de la Alcazaba se desarrolló en la muralla norte del mismo, tanto intramuros como extramuros, así como en su entorno más próximo, donde se localiza un aljibe de cronología medieval (Malpica, 2002, pp. 82-83) todavía en uso y restaurado en la primera mitad del siglo XX (Torres, 1969, pp. 69-94). Estuvo motivada por el proyecto de consolidación y restauración del baluarte, llevándose a cabo una actuación integral: seguimiento arqueológico, lectura estratigráfica mural del lienzo norte de la muralla y excavación de sondeos arqueológicos. Se presentan a continuación, de forma muy breve, los resultados de la intervención arqueológica, insistiendo siempre en su carácter preliminar.

\section{El seguimiento arqueológico y el estudio de elementos emergentes}

En lo referente al seguimiento arqueológico, esta actividad se extendió temporalmente durante todo el transcurso de la obra. En este sentido, fue muy significativa la actuación en el adarve de la muralla y la plataforma artillera, permitiendo documentar su configuración primigenia. Se pudo así afinar la lectura estratigráfica mural, la cronología y las diferentes soluciones y materiales constructivos que presenta este sector.

Construido a finales del siglo XV, el baluarte está levantado en tapia de hormigón de cal, más rico en dicho aglutinante en los cajones inferiores, debido a la necesidad de dotarlos de una mayor solidez y aislamiento, mientras que las tapias superiores, que constituyen la mayor parte de la muralla, son más pobres en cal. Las tapias finalizan mediante juntas oblicuas, cuya posición permite determinar la dirección de la construcción. El sector analizado presenta siete troneras con algunas diferencias notables en cuanto a su posición, siendo las tres primeras muy similares y situadas a una misma altura, comenzando a disminuir de cota a partir de la tronera 4 , en relación a un cambio en la cimentación de la muralla, como se verá.
A pesar de adscribirse a un único momento, esta cerca no es una estructura homogénea. Este desfase se aprecia claramente en la cimentación de la muralla. Los dos tercios más orientales asientan directamente sobre la formación Alhambra (denominación que damos a la base geológica) someramente trabajada y nivelada, para alcanzar un plano horizontal que permitiese asentar los tapiales, mientras que el tercio oeste fue cimentado mediante una zarpa tras excavar una zanja en la roca. Podría pensarse en la existencia de dos grandes fases históricas y constructivas, e incluso que la obra moderna amortiza una supuesta construcción medieval. No obstante, un análisis detallado descarta esta hipótesis. Tanto la muralla norte como el flanco este y la batería se adscriben a un único momento, finales del siglo XV. Las diferencias que se observan en la cimentación más bien parecen estar en relación con la topografía y/o a un cambio en el proyecto original por diferentes causas, entre ellas está, seguramente, la rapidez con la que se construyeron estos baluartes y a los altibajos presupuestarios, rastreable en la documentación de archivo (AGS, CMC, leg. 140; Vilar, 2007) Finalmente, la muralla del baluarte remata en un pretil de mampostería en cajones. En lo que respecta al parapeto coetáneo al baluarte, está perdido casi en su totalidad, a excepción de un único punto, en el perímetro curvo. Pero en su mayoría fue reconstruido en una fecha muy posterior y todavía por determinar. Parece ser coetáneo a la construcción de la gran losa de hormigón, pues esta se encofró apoyándose en dicho parapeto. Esta obra, la modificación más significativa que se efectuó en el baluarte, cambió seriamente la configuración de la zona, cegándose buena parte de las troneras.

\section{La excavación arqueológica}

La actuación más significativa fue la excavación de cuatro sondeos arqueológicos. Tenían por objeto buscar la base de los muros y aportar información de cara a la restauración, pero los resultados pronto superaron estas expectativas. $\mathrm{Al}$ interior se planteó el Sondeo 1, que abarcaba la mitad oeste de a muralla, siendo ampliado bajo la plataforma artillera. Al exterior, el Sondeo 2 comprendía toda la longitud del lienzo, 
desde la Torre de los Hidalgos, y parte del perímetro curvo de la plataforma artillera, dadas sus dimensiones, se dividió en tres sectores (A, B y C, de este a oeste). El Sondeo 3 se ubicó en el extremo suroeste de la batería. Finalmente, el Sondeo 4 se localizaba al oeste del aljibe exterior, todavía en uso, y la cara norte del tambor del baluarte.

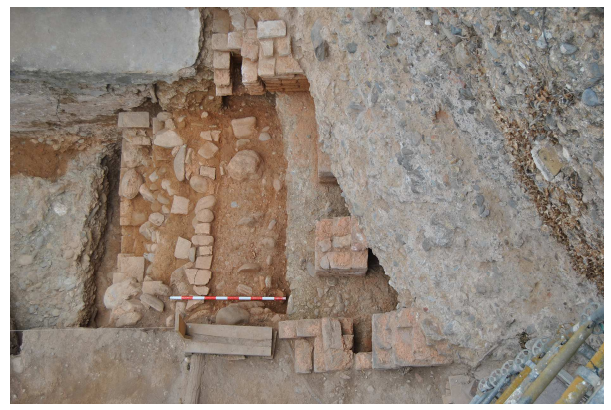

Fig. 7. Sondeo 4 (Teresa Koffler Urbano, 2019).

Los resultados obtenidos gracias a la excavación de estos sondeos han sido dispares, localizándose los hallazgos más interesantes en la zona noroeste, esto es, bajo la batería artillera y en torno al aljibe exterior (Sondeo 1 ampliación, Sondeo 2.C y Sondeo 4). Se ha sacado a la luz un edificio de cierta entidad, de uso relacionado con el agua y de cronología medieval, sobre el que se levanta el baluarte moderno. Este conjunto se extendería al oeste y sur del mencionado depósito, sin duda relacionado con el mismo. De este se ha documentado la entrada original de agua, situada en el muro de cierre meridional. Se realizaba mediante una canalización de atanores, con dirección y pendiente surnorte (Sondeo 2.C). Al oeste del aljibe (Sondeo 4) se localizaron los restos de lo que a todas luces es un hipocausto (Fig. 7) formado por pilares de ladrillo y tabiques del mismo material, en los que se abrían al menos dos posibles chimeneas. Estas estructuras están perfectamente alineadas y orientadas, presentando unas medidas idénticas. Se asientan sobre una plataforma muy homogénea de la formación Alhambra, que fue nivelada. En este sondeo, además, se localizó una potente estructura de mampostería encintada que se apoyaba en los muros oeste y sur del aljibe, parece que, como continuación del segundo, y con la misma orientación. Queda por definir su cronología, pues parece ser posterior al hipocausto, tal vez de época nazarí, lo que podía indicar una reutilización de los elementos previos con otra funcionalidad, o incluso tratarse de una obra ya moderna. Las estructuras halladas en el interior (Sondeo 1 ampliación) vienen a corroborar la importancia de los hallazgos (Fig. 8) pero también aportan una mayor complejidad de cara a su interpretación, tanto funcional como cronológica, pues el análisis estratigráfico pone de manifiesto la posible existencia de varias fases medievales.

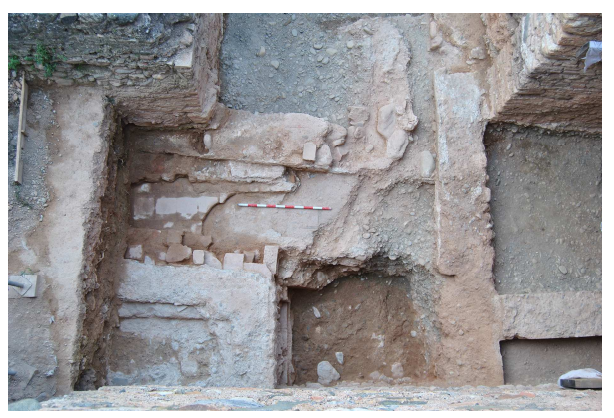

Fig. 8. Ampliación del Sondeo 1 (Teresa Koffler Urbano, 2019).

Se trata de una serie de muros de tapia con unas características y medidas muy similares, todos ellos presentan una base de hiladas de mampostería, dispuestas sobre la formación Alhambra para regularizar y aislar, sobre ella se levantaba el alzado de tapia. Llevan una orientación bien norte-sur, bien este-oeste, formando estancias de planta rectangular, habiéndose documentado al menos dos, aunque no se ha podido localizar ninguna completa. Todo este complejo parece bascular en torno a una potente estructura de hormigón de cal (Sondeo 1 ampliación y Sondeo 2.C). De planta rectangular y orientación norte-sur, podría corresponder a una pileta o pequeña alberca, y conserva embutidas dos pequeñas tuberías de plomo, una en la cara norte y otra en la sur. Se levanta sobre una estructura de ladrillo y losas de barro, dispuestas formando canalizaciones y de las que se han hallado al menos tres (Sondeo 2.C), de similar factura es 
un canal de losas de barro y ladrillo, con dirección y pendiente este-oeste (Sondeo 1 ampliación) documentada junto a la cara sur de esta supuesta alberca o pileta.

\section{Conclusiones}

Todo parece indicar que los restos hallados gracias a la excavación arqueológica corresponden a un baño, que tal vez formase parte de un conjunto más amplio cuya entidad está por definir, bien religioso, bien palatino o incluso de otro tipo. No obstante, hay que insistir en que se trata de unas conclusiones preliminares de carácter provisional, todavía en fase de investigación. De lo que no cabe duda es de la importancia de los hallazgos, puesto que no se tenía constancia, ni material ni documental. Existen unas muy vagas referencias textuales en la primera documentación castellana de finales del siglo XV (AGS, CMC, leg. 140; Malpica, Bermúdez, 1995, pp. 285-314; Vilar, 2007). En ella se menciona un "baluarte de la mezquita sobre el Darro", todavía no identificado con precisión y sobre cuya ubicación existen dudas (Malpica, Bermúdez, 1995, pp. 285-314) pero es muy plausible que se trate del actual Baluarte de la Alcazaba (Vilar, 2007). De esta manera, el topónimo, siempre con mucha precaución, podría hacer referencia a la funcionalidad de los restos exhumados. En lo que respecta a la cronología de este complejo, el análisis estratigráfico pone claramente de manifiesto que los restos son medievales, puesto que el baluarte se levanta directamente sobre los mismos. Acerca del origen del sistema defensivo no hay dudas gracias a la información documental y al análisis mural, es bien conocido que se edificó a finales del siglo $\mathrm{XV}$, inmediatamente después de la conquista cristiana. Más dificultades presenta el discernir las fases dentro de cada etapa, medieval y moderna, las cuales, sin duda, existieron. Como hipótesis preliminar, se han identificado al menos dos fases medievales, todavía por afinar. Con muchas reservas, la fase fundacional parece ser prenazarí, a la que corresponden la mayoría de las estructuras documentadas, amortizándose parcialmente ya en un momento posterior, esto es, en época nazarí, seguramente con otra funcionalidad. La construcción del baluarte a finales del siglo XV anuló este complejo, conservándose únicamente en uso el aljibe exterior.

Las obras han servido para cumplir los fines propuestos: interrumpir el proceso de deterioro que sufría la zona, mejorando su estado y aumentando el conocimiento. El conjunto de trabajos representa la complejidad de una restauración, donde la interdisciplinariedad y el trabajo en equipo resultan imprescindibles.

\section{Bibliography}

Arántegui y Sanz, J. (1891). Apuntes históricos sobre la Artillería Española en la primera mitad del siglo XVI, Imprenta del Cuerpo de Artillería, Madrid.

Cobos Guerra, F., coord. (2004). La artillería de los Reyes Católicos, Junta de Castilla y León.

Fernández y González, F. (1866, 2005). Estudio social y político de los Mudéjares de Castilla, Maxtor Ed., Valladolid. Malpica Cuello, A. (2002). La Alhambra de Granada. Un estudio arqueológico, Universidad de Granada Ed., Granada.

Malpica Cuello, A.; Bermúdez López, J. (1995). "Transformaciones cristianas en la Alhambra", in Acculturazione e mutamenti. Prospettivenell'Arqueologiamedievale del Mediterraneo, All'Insegna del Giglio Ed., Florencia, pp. 285-314.

Torres Balbás, L. (1969). "Diario de obras en la Alhambra. 1930-1936”, Cuadernos de la Alhambra, 5, pp. 69-94.

Vilar Sánchez, J.A. (2007). Los Reyes Católicos en la Alhambra, Comares Ed., Granada. 